\title{
Study on Factors Affecting Residual Magnetism of Phase Selection of Extreme High Voltage Transformer and Its Calculation Method
}

\author{
Teng Wentao ${ }^{1}$, Xiang Zutao ${ }^{1}$, Zheng Bin ${ }^{1}$, Song Xiuyou ${ }^{2}$, Zhang Yuanyuan ${ }^{1}$, Li Kuan ${ }^{3}$ \\ ${ }^{1}$ Power System Department, China Electric Power Research Institute, Beijing, China \\ ${ }^{2}$ School of Electrical Engineering, Beijing Jiaotong University, Beijing, China \\ ${ }^{3}$ Shandong Electric Power Research Institute, Jinan, China
}

Email address:

tengwentao@epri.sgcc.com.cn (Teng Wentao),xzt@epri.sgcc.com.cn (Xiang Zutao)

\section{To cite this article:}

Teng Wentao, Xiang Zutao, Zheng Bin, Song Xiuyou, Zhang Yuanyuan, Li Kuan. Study on Factors Affecting Residual Magnetism of Phase Selection of Extreme High Voltage Transformer and Its Calculation Method. Journal of Electrical and Electronic Engineering.

Vol. 7, No. 2, 2019, pp. 57-63. doi: 10.11648/j.jeee.20190702.15

Received: April 20, 2019; Accepted: May 27, 2019; Published: June 15, 2019

\begin{abstract}
As one of the core components in the extreme high voltage power grid, the extreme high voltage transformer has a large rated capacity, and the level of magnetizing inrush current generated by the air-to-air transformer is high. At the same time, a large amount of harmonics are injected into the power grid. In severe cases, a long duration and a slow decay can be generated. The voltage has an adverse effect on the near-area grid and equipment (especially power electronics). In order to further improve the safety and reliability of systems and equipment, effective measures need to be taken to suppress them. At present, the measures that can be used to limit the main transformer closing current include the circuit breaker installation closing resistor and the phase selection closing technology. However, since the closing resistance does not exceed $10 \mathrm{~ms}$, it limits the main transformer closing excitation current, harmonics and over The effect of voltage is relatively limited, and the conventional phase-selection closing technology cannot consider the influence of transformer remanence, and its limiting effect is also not ideal. Foreign research and the use of phase-selection closing technology that takes residual magnetism in some ultra-high-pressure projects to solve the problem of air-to-air inrush current has gained some experience. In theory, this measure is expected to solve the extreme high voltage large-capacity transformer very ideally. Inrush current, harmonics, waveform distortion and overvoltage caused by closing. In this paper, the influencing factors of remanence of extreme high voltage transformers in phase-selective switching technology are studied. According to the research results, the specific scheme of voltage integral to measure residual magnetism is established.
\end{abstract}

Keywords: Extreme High Voltage Transformer, Residual Magnetism, Option Switching, Voltage Integral Calculation Method

\section{Introduction}

Phase-selective switching technology, also known as phase control technology or synchronous switching technology, was first proposed in the 1970s [1], essentially controlling the opening and closing time of the circuit breaker by phase separation (ie, the initial phase angle of the system voltage or current during closing) In order to reduce the inrush current and overvoltage of the switch closing operation, eliminate the re-ignition overvoltage of the trip, improve the breaking capacity of the circuit breaker and the reliability and economy of the system [2]. With the advancement of modern electrical manufacturing levels and microelectronics automation technology, this technology has been applied to many aspects of transmission and distribution systems.

The prevalence of residual magnetism in transformers makes it difficult to eliminate the shunt magnetizing inrush current by conventional phase-selection closing technology [3]. When the breaker of the transformer is turned off instantaneously, the magnetic flux in the iron core first drops immediately, and then enters the equilibrium state after a 
brief magnetic aftereffect phase transition [4-5]. After that, the residual magnetization changes with time is not obvious, if the field temperature is not guaranteed It will mutate more than the Curie point of the iron core and the electromagnetic noise at the scene is small, which is not enough to affect the change of the magnetic field. It can be considered that the residual magnetism hardly changes with time, and the residual magnetism will not disappear naturally in the iron core for a long time [6].

The phase selection closing technology is adopted to control the closing time of the circuit breaker by phase selection, and the transformer is input into the transformer when the pre-induced magnetic flux in the iron core is equal to the residual magnetism [7], so that the core saturation can be avoided, thereby effectively suppressing the magnitude of the magnetizing inrush current. This technology is more reliable and economical than limiting inrush currents based on closing resistors and shunt capacitors.

At present, there are many studies on the measurement of core remanence after transformer opening at home and abroad, but there is still no mature and effective method. Various theories, models, data and experiments need to be further studied. Therefore, it is convenient to study. Effective remanence measurement methods are of great significance.

The existing methods of remanence analysis are mainly divided into the following types:

(1) Empirical estimation of residual magnetism. It is generally believed that the remanence after the test run is in the range of $20 \%$ to $80 \%$ saturation flux. The empirical estimation method of remanence can provide a reference for transformer manufacturers to carry out the factory transformer test, but this method can not obtain accurate remanence value [8].

(2) Calculation method of core remanence [9]. The core remanence is calculated by establishing the equivalent model of the transformer. The reliability and accuracy of the equivalent model of the transformer lies in the modeling of the core. The calculated results often have large errors, which are not applicable and widely used.

(3) Pre-magnetization method of residual magnetism [10]. By applying an external power source to the core, it is excited from the original remanence value to a known remanence, and then a phase selection closing operation is performed on the basis of the known remanence to suppress the magnetizing inrush current. For large transformers, the equipment required for the pre-magnetization method is expensive, and a large current is required to generate magnetic flux, which is bound to affect the transformer itself.

(4) Direct measurement method of residual magnetism [11]. The residual magnetism can be measured by a Gauss meter or the like, but this method can only test the magnetic properties of the surface of the ferromagnetic material, which is not accurate.

(5) Indirect measurement method of residual magnetism. The peak value of the magnetizing inrush current is obtained by the transformer energization test, and the value of the remanence is obtained based on the peak value. This method can only obtain the residual magnetic value after the transformer is closed, and can not calculate the residual magnetic value before closing, which limits the feasibility of the method.

(6) Voltage integration method [12]. The method calculates residual magnetization based on the transformer inlet voltage, which is most commonly used. The voltage transformer is used to measure the voltage at the end of the transformer winding, and then the integral method is used to calculate the flux linkage. After the transformer inlet voltage or winding current disappears (usually several hundred ms after the trip), the flux linkage is stable. This flux linkage value is the remanence.

In this paper, the factors affecting the remanence of the transformer, such as the opening time, the equivalent capacitance of the circuit and the equalizing capacitance of the circuit breaker, are theoretically analyzed. Several variables are simulated by ATP-EMTP software, and finally based on the research results. The implementation of voltage integration to measure residual magnetism.

\section{Study on the Factors Affecting Remanence}

\subsection{Opening Time}

Theoretically, the residual magnetization can be calculated by integrating the transformer winding voltage. The phase angle of the residual magnetic backward voltage is $90^{\circ}$, and the measured voltage value after the transformer is opened can be calculated according to the following formula (1).

$$
\phi_{r}=\frac{1}{N} \int_{t_{0}}^{t_{1}} u d t
$$

If the power supply voltage is:

$$
u=U_{m} \sin (\omega t)
$$

Can be calculated:

$$
\phi_{r}=-\phi_{m} \cos \left(\omega t_{1}\right)=-\frac{U_{m}}{N \cdot \omega} \cos \left(\omega t_{1}\right)
$$

The formula of remanence after the breaking of the three-phase transformer can be extended from equation (3):

$$
\left\{\begin{array}{l}
\phi_{r A}=-\phi_{m} \cos \left(\omega t_{1 A}\right) \\
\phi_{r B}=-\phi_{m} \cos \left(\omega t_{1 B}+120\right) \\
\phi_{r C}=-\phi_{m} \cos \left(\omega t_{1 C}-120\right)
\end{array}\right.
$$

$\Phi r$-The amount of residual magnetism in the transformer; $\Phi m$-The peak value of the magnetic flux;

$N$-The number of turns of the winding; $u$-voltage;

$t_{0}$-Initial moment 
$t_{1}$-The breaking moment of the transformer;

$\omega$-Power angle frequency.

Figure 1 shows the minimum and maximum remanence of the transformer after the transformer is turned off. The figure shows the relationship between the breaking phase angle and the residual magnetism of the transformer after the last time the no-load transformer was opened. As shown in Figure 1 (a), the $\mathrm{B}$ and $\mathrm{C}$ phases are opened at the same phase of the two-phase magnetic flux. At this time, due to the action of the A phase, the magnetic fluxes of the $\mathrm{B}$ and $\mathrm{C}$ phases are gradually less, after $1 / 4$ cycle three. The remanence of the phase is at least zero, and the phase A is broken at this time. As shown in Figure 1 (b), the A and B phases are first separated at the same time when the forward voltage is equal. After that, the phase $\mathrm{C}$ is zero at the phase voltage (when the phase $\mathrm{C}$ flux is the largest), and the residual magnetism is the most serious. In the figure, the ideal situation is neglected by other factors such as the dynamic characteristics of the iron core. In practice, the residual magnetism is almost impossible to achieve, and the actual three-phase remanence is complicated. Commonly used to reduce the remanence of the transformer core includes DC degaussing, raising the core temperature and selecting a new soft magnetic material with less coercive force.

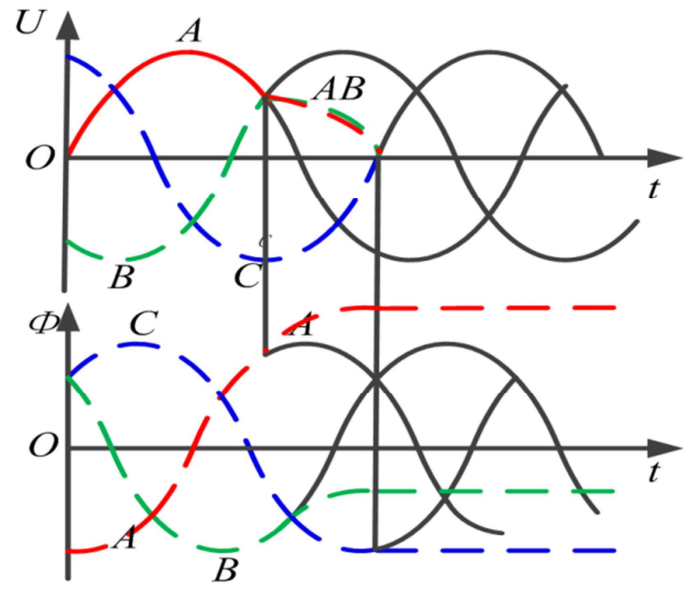

(a). Minimum remanence.

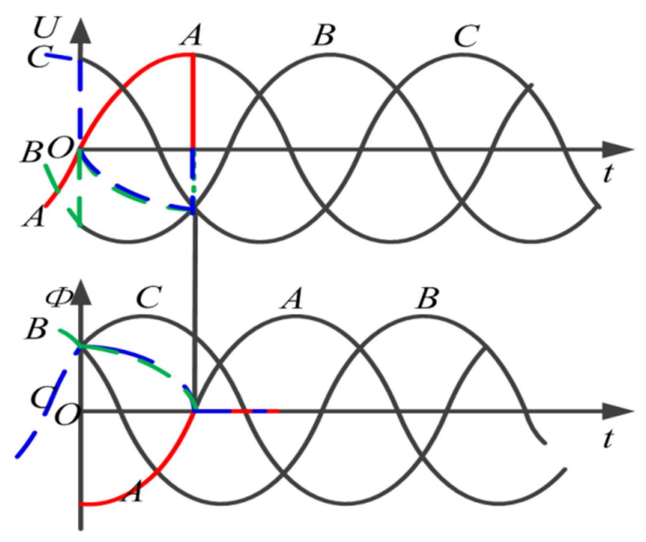

(b). Maximum remanence.

Figure 1. Principle of residual magnetization after transformer breaking.
In theory, the remanence can be calculated according to the opening moment, but in the actual project, the remanence is also affected by other influencing factors, making the calculation of residual magnetism complicated.

\subsection{Circuit Equivalent Capacitance}

After the transformer exits the operation, the equivalent capacitance in the circuit and the equivalent inductance of the transformer form an oscillating circuit. The system voltage and current are reduced to zero after the switching transient process, and the magnetic flux is stabilized in a certain determination after the switching transient process. value. The equivalent simplified circuit after opening is shown in Figure 2.

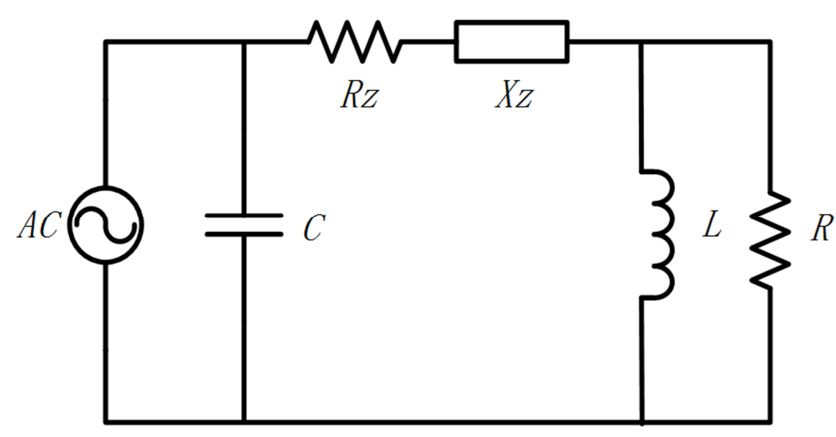

Figure 2. Equivalent simplified circuit diagram after opening.

$\mathrm{C}$ _ the sum of the equivalent capacitance of the transformer, the capacitance of the capacitor bank, the capacitance of the line, etc.;

$\mathrm{L}$ _ the equivalent nonlinear inductance of the transformer core;

$\mathrm{R}$ — the equivalent resistance of the transformer core;

$\mathrm{R}_{\mathrm{Z}}$ winding resistance;

$\mathrm{X}_{\mathrm{Z}} \longrightarrow$ leakage resistance.

The oscillation process after the opening is LC oscillation. Since the RZ is much smaller than the equivalent resistance of the transformer core, the $\mathrm{XZ}$ value is small and negligible, so the circuit can be simplified to the parallel RLC circuit. For the parallel RLC circuit, the oscillation characteristics can be described for:

$$
\begin{aligned}
& \omega_{0}=\frac{1}{\sqrt{L(i) C}} \\
& \alpha=\frac{1}{2 R C} \\
& \omega_{N}=\sqrt{\omega_{0}^{2}-\alpha^{2}} \\
& \xi=\frac{\alpha}{\omega_{0}}=\frac{1}{2 R} \sqrt{\frac{L(i)}{C}}
\end{aligned}
$$

$\omega_{0}$ - undamped resonant frequency;

$\alpha$ - damping coefficient; 
$\omega_{N}$ - natural frequency;

$\xi$ - Damping ratio ( $\xi<1$ for underdamped, $\xi=1$ for critical damping, $\xi=1$ for overdamped).

Since the value of the winding capacitance is large, the damping ratio of a large transformer is generally much smaller than 1, so the undamped resonant frequency can be considered to be approximately equal to the natural frequency. It can be seen from the above formula that the larger the equivalent capacitance value in the circuit, the smaller the damping coefficient, and the longer the transient process of the oscillation continues.

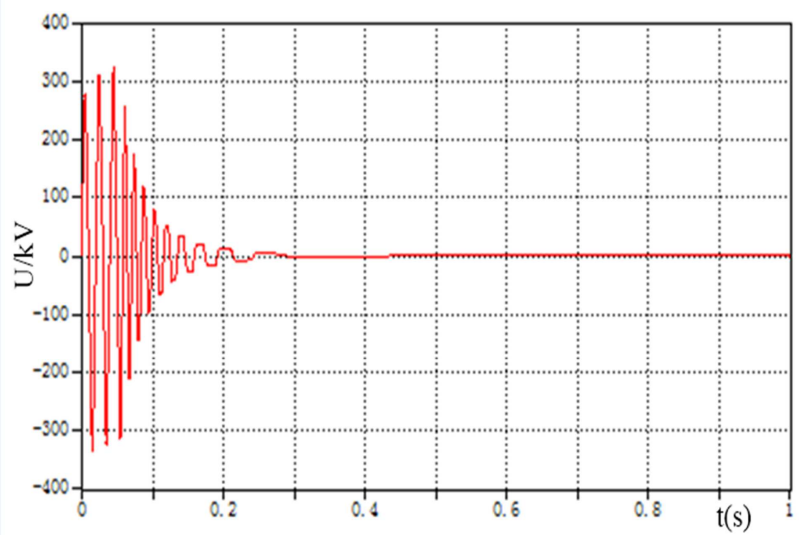

(a). Voltage waveform during oscillation $(\mathrm{C}=200 \mu \mathrm{F})$

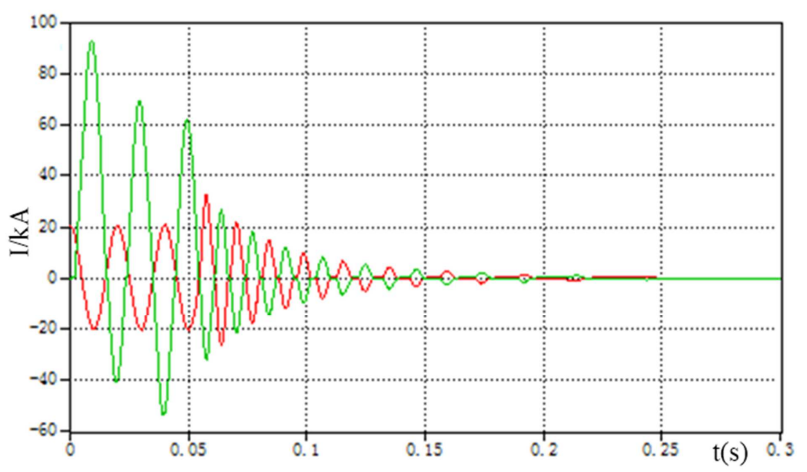

(b). Current waveform during oscillation $(\mathrm{C}=200 \mu \mathrm{F})$

Figure 3. Voltage and current waveform during oscillation.

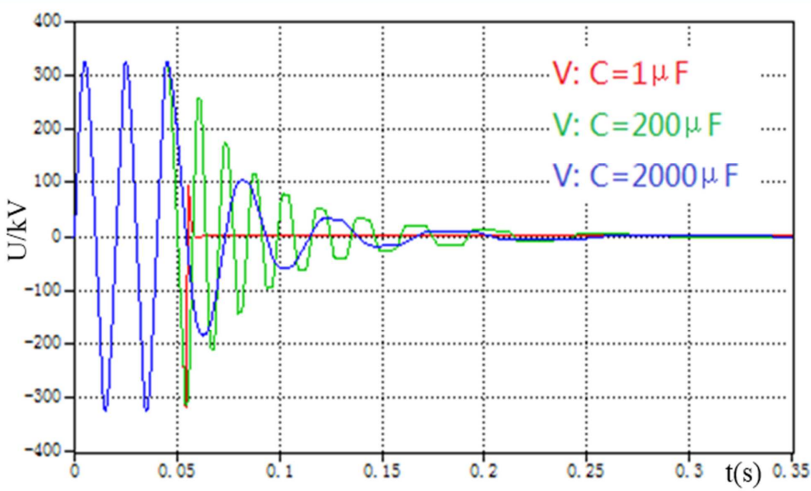

(a). Voltage oscillation process

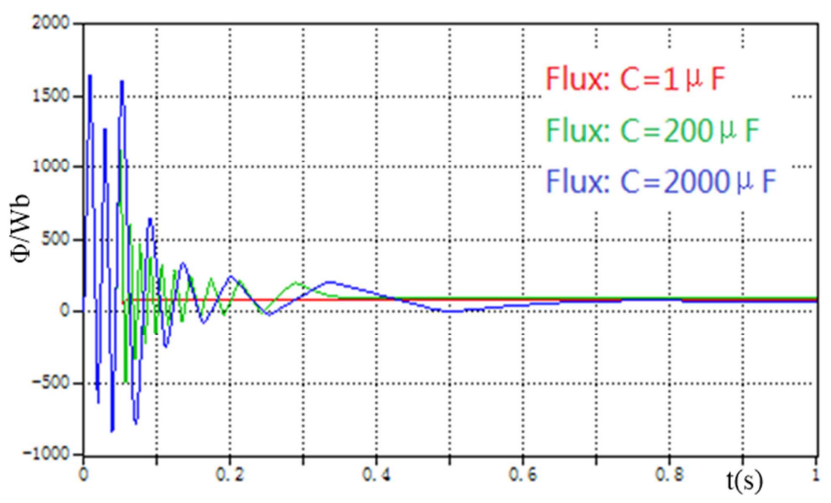

(b). Flux oscillation process

Figure 4. Voltage and flux oscillation process at different capacitance values.

In the ATP-EMTP, a simulation model is built according to the circuit of Figure 2, and a voltage integration module is added to the circuit to measure the magnetic flux. The voltage and current waveforms of the oscillating circuit collected during the oscillation process are shown in Figure 3. As can be seen from the figure, the voltage and current will oscillate after a period of time after opening, and eventually drop to zero. In order to simulate the influence of different equivalent capacitance values on remanence, the equivalent capacitance value is changed in the simulation model for multiple simulations, and the simulation results are displayed on the same interface. The voltage and flux oscillation process under different capacitance values are shown in the figure. As shown in Figure 4, as the equivalent capacitance increases, the duration of the oscillation process after the opening is gradually increased. If the re-magnetization is obtained by the voltage integration method widely used in engineering, the oscillation duration will directly affect the integral upper limit value of the voltage integral in the calculation of residual magnetism, which will affect the accuracy of the residual magnetization calculation.

\subsection{Circuit Breaker Equalizing Capacitor}

Modern high-voltage switches often use multiple fractures. In order to evenly distribute the voltage across the fractures between the contacts to ensure the arc-extinguishing capability of the switches, the equalization capacitors are often connected in parallel. After the transformer is cut off by a circuit breaker equipped with a voltage equalizing capacitor, the bus voltage on the power supply side is transmitted to the transformer side through the voltage equalization capacitive coupling, thereby affecting the residual magnetism of the transformer core.

Figure 5 shows the equivalent circuit of the no-load transformer breaking considering the equalizing capacitance of the circuit breaker. In the figure, $\mathrm{Cg}$ is the circuit breaker equalizing capacitor, $\mathrm{C}$ is the transformer equivalent to ground capacitance, $\mathrm{L}$ is the no-load transformer magnetizing inductance, and $\mathrm{R}$ is the equivalent resistance considering the eddy current loss and hysteresis loss of the core material. 


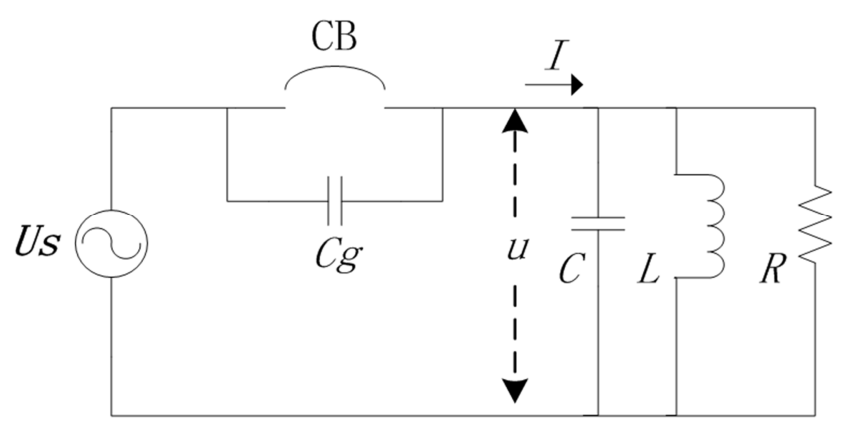

Figure 5. Breaking the equivalent circuit of the no-load transformer.

Set the system voltage when opening:

$$
u_{s}(t)=U_{s m} \sin \left(\omega t+\varphi_{s}\right)
$$

$U_{s m}$ - System voltage peak;

$\omega$ - System voltage angular frequency;

$\varphi_{s}$ - Phase angle of the voltage at the moment of opening.
After the circuit breaker is opened, the system power supply forms a path through the voltage equalizing capacitor and the transformer. When the circuit reaches steady state, the voltage across the transformer is:

$$
\left\{\begin{array}{l}
u(t)=U_{m} \sin \left(\omega t+\varphi_{0}\right) \\
\varphi_{0}=\varphi_{s}-\arctan \frac{\omega L}{\omega^{2} L R\left(C+C_{g}\right)-R} \\
U_{m}=\frac{C_{g}}{\sqrt{\left(C_{g}+C-\frac{1}{\omega^{2} L}\right)^{2}+\left(\frac{1}{\omega R}\right)^{2}}} U_{s m}
\end{array}\right.
$$

It can be obtained from equation (10). Under the influence of the voltage equalizing capacitor, there is still an alternating voltage at both ends of the transformer after the opening. Next, the influence of the alternating voltage on the residual magnetization is simulated.

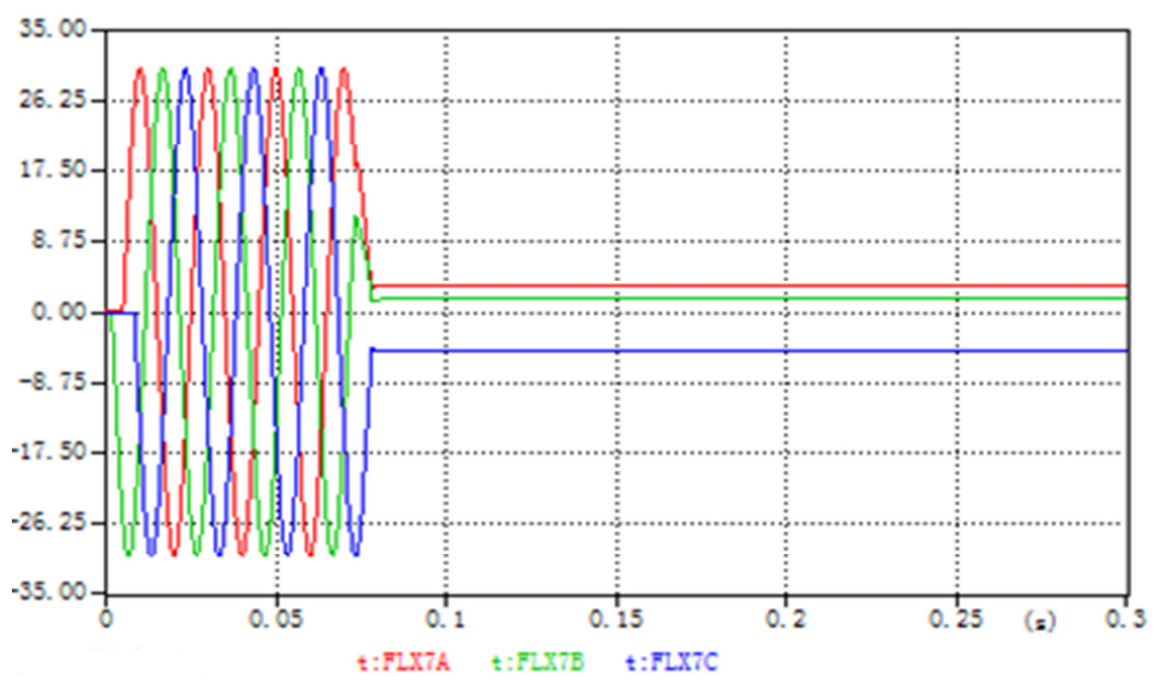

Figure 6. Flux waveform without averaging capacitor.

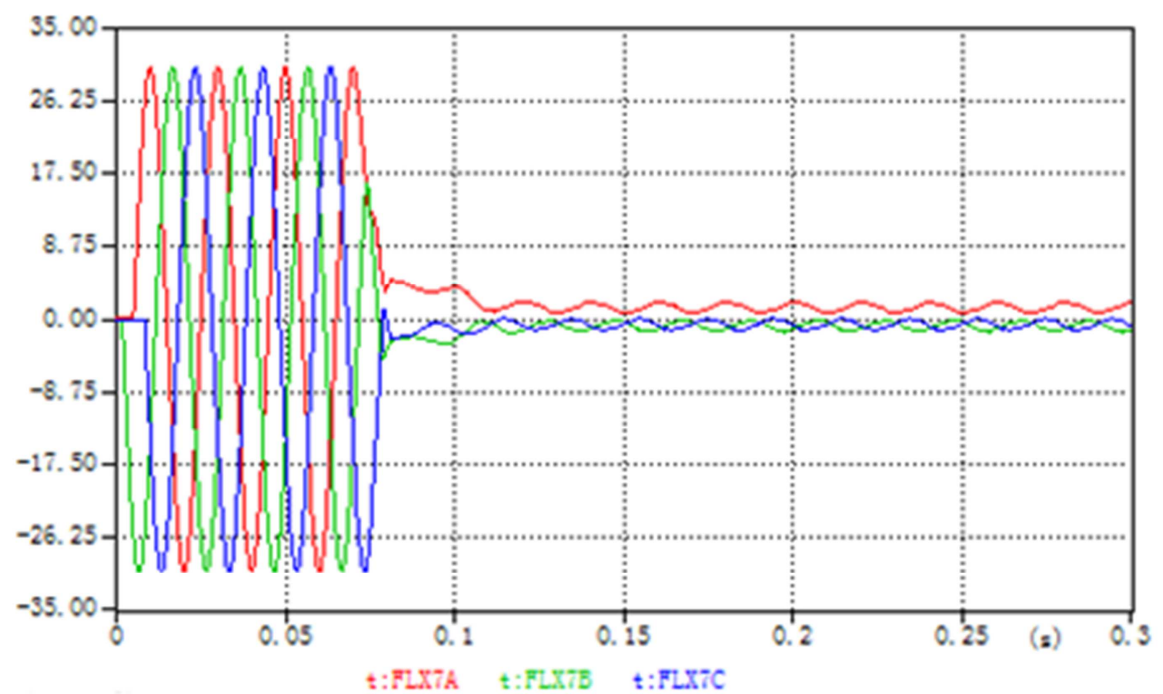

Figure 7. Residual magnetic waveform when there is a voltage equalizing capacitor. 


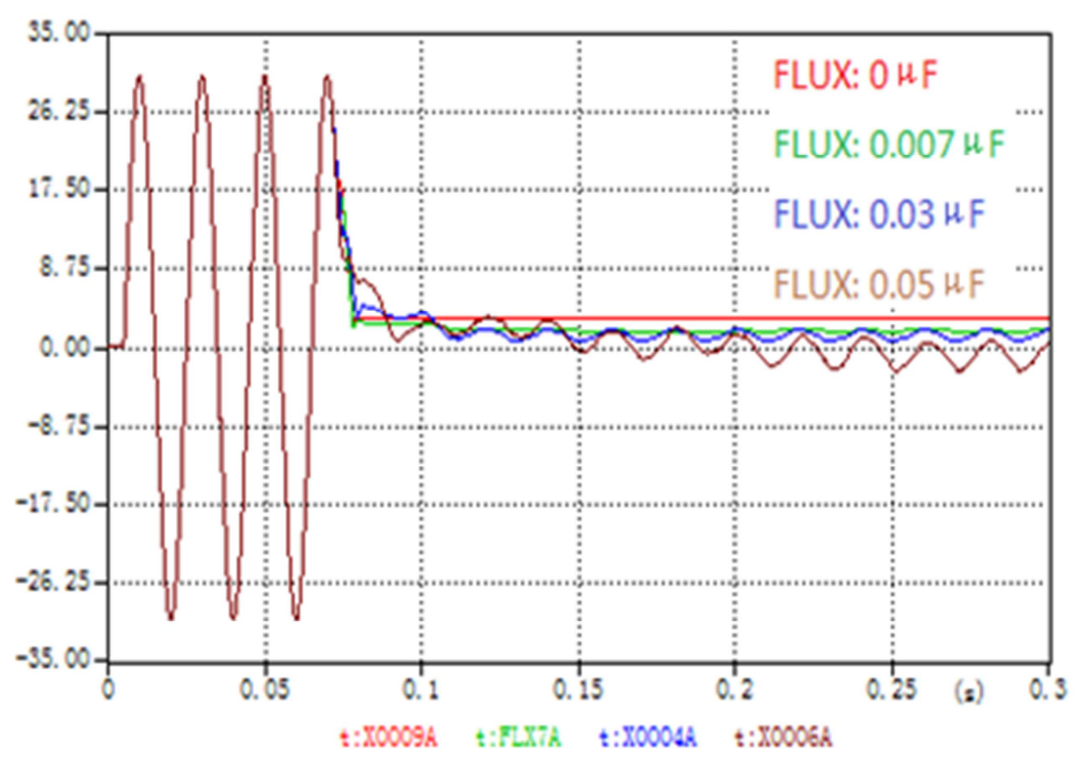

Figure 8. Residual magnetic waveform under different grading capacitance values.

It can be seen from the simulation results that after the transformer is cut off by the circuit breaker with the equalizing capacitor, the residual magnetization of the transformer core is no longer a stable stable value, but a periodic dynamic remanence; The larger the pressure capacitor, the lower the residual magnetization level of the transformer core after opening, and the existence of the voltage equalizing capacitor of the circuit breaker has the effect of weakening the core remanence after the transformer is opened.

\section{Method for Calculating Voltage Integral of Residual Magnetism}

In theory, the remanence can be obtained by integrating the voltage of the primary winding of the transformer, but the resulting error is often large, and the most important cause of the error is the inaccuracy of the upper limit of the voltage integration. Due to the characteristics of the core, the transformer winding capacitance and other capacitors in the circuit, and the transient recovery voltage of the circuit breaker, the residual magnetization can be stabilized for a period of time after the trip, so the voltage integral obtained at the moment of opening is obtained. Magnetic is not accurate. In order to make the voltage integration method more accurate, it is important to accurately obtain the upper limit of the voltage integration.

The calculation principle of residual magnetism is shown in Figure 10. It can be seen that the voltage of the primary winding of the transformer will not drop to zero immediately after the opening, but will gradually become zero after a period of attenuation oscillation, and the absolute value of the voltage is taken. And the peak value of each half wave after the opening is fitted, the voltage stabilization time can be obtained, thereby determining the upper limit of the voltage integration, and the difference between the magnetic flux value obtained by the obtained upper limit of the integral and the magnetic flux center value before the opening. It is the remanence.

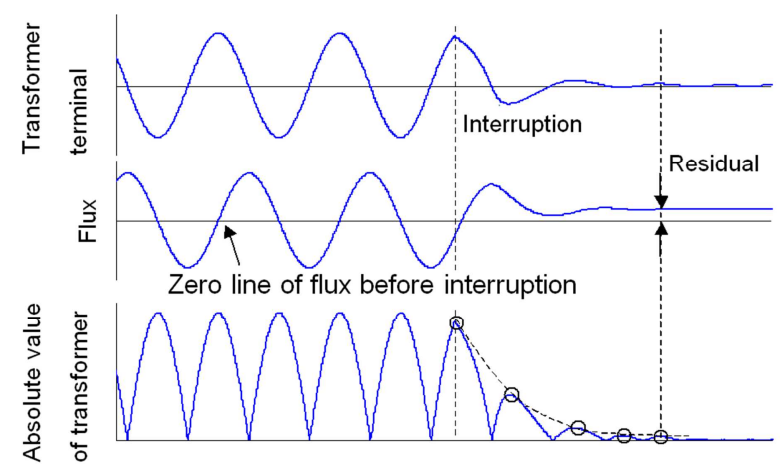

Figure 9. Residual magnetic measurement principle.

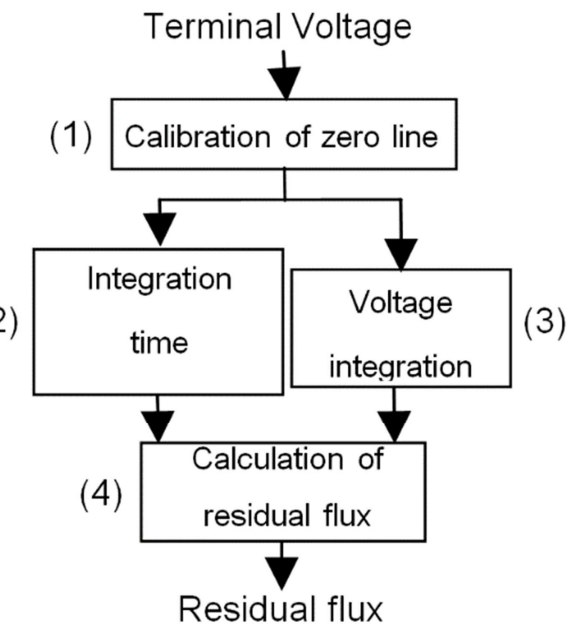

Figure 10. Residual magnetic measurement process.

The residual magnetic measurement steps are shown in Figure 10: 
(1) discretizing the voltage waveform to obtain discrete data corresponding to the waveform;

(2) Filtering processing, removing burrs in the waveform, etc., to make the waveform smoother;

(3) taking the voltage waveform as an absolute value, and extracting the peak value of each half wave of the voltage absolute value waveform as a basis for fitting;

(4) fitting the peak point after the opening by the least square method, thereby determining the voltage stability point, that is, the upper limit of the voltage integration;

(5) Numerical integration of the voltage, using the upper limit of the integral obtained by the fitting, to obtain a more accurate remanence value.

\section{Conclusion}

(1) Under ideal conditions, the residual magnetism of the three-phase transformer after breaking can be accurately calculated by voltage integration of the transformer winding, but in actual engineering, the residual magnetism is also affected by other influencing factors such as the dynamic characteristics of the iron core. The case of zero remanence is almost impossible to achieve.

(2) Through the simulation model, it is found that as the equivalent capacitance increases, the duration of the oscillation process increases gradually after opening. If the re-magnetization is obtained by the voltage integration method widely used in engineering, the oscillation duration will directly affect the integral upper limit value of the voltage integral in the calculation of residual magnetism, which will affect the accuracy of the residual magnetization calculation.

(3) After the transformer is cut off by the circuit breaker equipped with the equalizing capacitor, the residual magnetization of the transformer core is no longer a stable stable value, but a periodic dynamic remanence; the larger the equalizing capacitance of the circuit breaker broken in parallel The lower the residual magnetic level of the transformer core after opening, the presence of the voltage equalizing capacitor of the circuit breaker has a weakening effect on the core remanence after the transformer is opened.

(4) Through research, it is found that the most important cause of the error in the calculation method of voltage integral is that the upper limit of the integral is not accurate. In order to make the voltage integration method more accurate, it is important to accurately obtain the upper limit of the voltage integration. After the opening, the voltage of the primary winding of the transformer will not immediately drop to zero, but will gradually become zero after a period of fading oscillation. By taking the absolute value of the voltage and fitting the peak value of each half wave after opening, The voltage stabilization time can be obtained, thereby determining the upper limit of the voltage integration, and the difference between the magnetic flux value obtained by the obtained upper limit of the integral and the magnetic flux center value before the opening is the remanence.

\section{References}

[1] Niu Shuaijie, Zhao Lihua, Chen Ling, et al. Research on Residual Magnetic Resonance Detection Method Based on Time-Current Curve $[\mathrm{J}]$. Electric Measuring \& Instrumentation, 2017 (07): 71-75+87.

[2] Zhou Jianping, Luo Jian, ZHOU Jian-ping, et al. An Estimation Method for Residual Magnetism of Transformer Core [J]. Thermal Power Generation, 2010, 39 (3): 61-64.

[3] Xing Yunmin, Luo Jian, Zhou Jianping, et al. Residual Magnet Estimation of Transformer Core [J]. Power System Technology, 2011 (2): 169-172.

[4] He Yuan, Li Xin, Luo Jian. Research on Identification of Transformer Magnetizing Inductance Using Hysteresis Loop [J]. Power System Protection and Control, 2013 (14).

[5] Zhang Xiaojie, Yang Bo, Chen Lei. Research on Calculation Method of Residual Magnetism of Transformer Based on Hysteresis Loop [J]. 1997, 52 (10): 11-13.

[6] Yang Yuyan, Wang Bao. Transformer Model Considering Hysteresis Based on PSCAD V4.6 [J]. Guangdong Electric Power, 2017, 30 (6): 67-72.

[7] Qi Guozheng. Research on Transformer Magnetizing Inrush Current Suppression Technology [D]. 2017.

[8] Ge Wenqi, Wang Youhua, Chen Xueguang, et al. Measurement and weakening method of core remanence in power transformers $[\mathrm{J}]$. Transactions of China Electrotechnical Society, 2015, 30 (16): 10-16.

[9] Huang Jin, Liang Zhaoting. Selection and correlation technology based on residual magnetic measurement $[\mathrm{J}]$. Electrical Apparatus and Energy Efficiency Management Technology, 2011 (9): 10-13.

[10] Wang Yang. Research on Remanence Prediction of Transformer Core [D]. Shandong University, 2017.

[11] Huang Jin. Research on the selection and correlation technology of no-load transformers [D]. 2010.

[12] Ni Haimiao. Research on the method of suppressing the inrush current of the no-load transformer based on phase selection control [D]. 2016. 